position fields coincide and are cyclic. The field $\bar{L}$ is then equivalent to a subfield of $\bar{K}^{\prime}$; without loss of generality we may suppose $\bar{K}^{\prime}>\bar{L} \geqq \bar{K}$. The degree $[\bar{L}: \bar{K}]=\bar{m}$ is a divisor of $m$. Consequently $\left[Z_{n} \bar{L}: \bar{K}\right]=\left[Z_{n} \bar{L}: \bar{L}\right][\bar{L}: \bar{K}]=n \bar{m}$. By the Galois theory there is then for every integer $n$ an extension $Z_{n}^{*}$ of degree $n$ over $\bar{K}$. The defining equation $f^{*}(x)=0$ of $Z_{n}^{*} / \bar{K}$ now may be approximated by an irreducible equation $f(x)=0$ of degree $n$ with coefficients in $K$ so that $Z_{n}^{*}$ is generated by the roots of $f(x)=0$. The root field of $f(x)=0$ over $K$ is the cyclic extension $Z_{n}^{\prime}$ of degree $n$ over $K$. Hence $Z_{n}^{*}=Z_{n}^{\prime} \bar{K}$ for all $n$, contrary to the assumption that $K$ is not relatively complete with respect to any rank one valuation.

HARVARD UNIVERSITY AND

University OF CHICAGO

\title{
A DIFFERENTIAL GEOMETRY PROBLEM USING TENSOR ANALYSIS
}

ATHERTON H. SPRAGUE

1. Introduction. The problem at hand was worked out in attempting to apply tensors to a much more general problem in classical differential geometry. The results obtained in a general coordinate system reduce readily to classical results of Eisenhart. An interesting interpretation of Christoffel symbols appears.

2. $R$ net. A rectilinear congruence in 3 -space is called a $W$-congruence if the asymptotic lines on the two focal surfaces correspond. If the tangents to both families of curves of a conjugate net on a surface form $W$-congruences the net is called an $R$ net. ${ }^{1}$ We derive the analytic conditions that must obtain in order that a given conjugate net on a surface shall be an $R$ net.

3. Equations for an $R$ net. Let $S_{1}$ be one focal surface of a $W$-congruence, the vector equation of the surface being

$$
z_{1}^{\alpha}=z_{1}^{\alpha}\left(x^{i}\right), \quad \alpha=1,2,3 ; i=1,2 .
$$

Received by the editors November 11, 1941.

${ }^{1}$ Tzitzeica, Comptes Rendus de l'Académie des Sciences, Paris, vol. 152 (1911), p. 1077. 
Let $S_{2}$ be the other focal surface of the congruence with vector $z_{2}^{\alpha}$ so that we have

$$
z_{2}^{\alpha}=z_{1}^{\alpha}+\rho_{1} \xi_{1}^{\alpha}
$$

where $\xi_{1}^{\alpha}$ is a unit vector tangent to $S_{1}$, and $\rho_{1}$ is an invariant.

Then if $\lambda_{1}^{i}$ are the components of this vector in the $x$ 's we have

$$
\xi_{1}^{\alpha}=z_{1 /, i}^{\alpha} \lambda_{1 /}^{i}
$$

where in this case

$$
\underset{z_{1 /, i}^{\alpha}}{\stackrel{\alpha}{\alpha}}=\frac{\partial z_{1}^{\alpha}}{\partial x^{i}}
$$

since $z_{1}^{\alpha}$ being an invariant for a transformation of coordinates in the $x$ 's, the ordinary derivative of $z_{1}^{\alpha}$ with respect to $x^{i}$ is the same as the covariant derivative with respect to $g_{i j}$, the fundamental tensor of $S_{1}$. Then substituting (3.3) in (3.2) we have

$$
z_{2}^{\alpha}=z_{1}^{\alpha}+\rho_{1} z_{1 /, i}^{\alpha} \lambda_{1 /}^{i}
$$

Similarly by the property of focal surfaces we have

$$
z_{1}^{\alpha}=z_{2}^{\alpha}+\underset{\rho_{2} z_{2 /, i}^{\alpha} \lambda_{2 /}^{i}}{\alpha}
$$

where $\rho_{2}$ is an invariant, and $\lambda_{2 /}^{i}$ is a unit vector tangent to $S_{2}$.

Adding (3.4) and (3.5) we have

$$
\rho_{1} \lambda_{1 / z_{1 /, i}^{i}}^{i}+\rho_{2} \lambda_{2 / z_{2 /, i}}^{i}=0 .
$$

From (3.6) we have

$$
\begin{aligned}
\rho_{1} & =\bar{e} \rho_{2}, \\
\lambda_{1 / z_{1 /, i}^{\alpha}} & =e \lambda_{2 / z_{2 /, i}}^{i}
\end{aligned}
$$

where $\bar{e}=1$ if $e=-1$, and $\bar{e}=-1$ if $e=1$, and conversely.

We differentiate (3.4) covariantly and have

$$
\stackrel{\alpha}{z_{2 /, k}}=\stackrel{\alpha}{z_{1 /, k}}+\rho_{1 /, k} \lambda_{1 / z_{1 /, i}^{i}}^{i}+\rho_{1} \lambda_{1 /, k}^{i} \underset{1 /, i}{\alpha}+\rho_{1} \lambda_{1 / z_{1 /, i k}}^{i} \underset{\alpha}{\alpha}
$$

Let $\eta_{1}^{\alpha}$ be the unit normal to $S_{1}$.

We multiply (3.7) by $\lambda_{2 /}^{k}$, sum for $k$, multiply by $\eta_{1}^{\alpha}$, sum for $\alpha$ and we have

$$
0=\rho_{1} b_{1 / i j} \lambda_{1 / \lambda}^{i} \lambda_{2 /}^{j}
$$

the first three terms on the right vanishing because $\eta_{1}^{\alpha}$ is perpendicular to $S_{1}$, the term on the left vanishing because of this fact and (3.6). 
and the last term becoming $\rho_{1} \eta_{1}^{\alpha} \cdot z_{i, i_{j}}^{\alpha} \lambda_{1 /}^{i} \lambda_{2 /}^{j}$ the second factor of which is denoted by $b_{1 / i j} \lambda_{1 /}^{i} \lambda_{2 / \circ^{j}}^{2}$

Hence the directions $\lambda_{1 /}^{i}$ and $\lambda_{2 /}^{j}$ are conjugate on $S_{1}{ }^{3}$

Similarly they are conjugate on $S_{2}$.

We next differentiate (3.7) covariantly with respect to $g_{i j}$, the fundamental tensor of $S_{1}$,

$$
\begin{aligned}
\frac{\partial^{2} z_{2}^{\alpha}}{\partial x^{k} \partial x^{j}} & -z_{2 /, m}^{\alpha}\left\{\begin{array}{l}
m \\
k j
\end{array}\right\}^{\rho_{i j}}=z_{1 /, k j}^{a}+\rho_{1 /, k j} \lambda_{1 / z_{1 /, i}^{i}}^{\alpha}+\rho_{1 /, k} \lambda_{1 /, j}^{i} z_{1 /, i}^{\alpha} \\
& +\rho_{1 /, k} \lambda_{1 / z_{1 /, i j}}^{i}+\rho_{1 /, j}^{\alpha} \lambda_{1 /, k}^{i} z_{1 /, 2}^{\alpha}+\rho_{1} \lambda_{1 /, k j}^{i} z_{1 /, i}^{\alpha}+\rho_{1} \lambda_{1 /, k}^{i} z_{1 /, i j}^{\alpha} \\
& +\rho_{1 /, j} \lambda_{1 / z_{1 /, i k}}^{i}+\rho_{1} \lambda_{1 /, j}^{i} z_{1 /, i k}^{\alpha}+\rho_{1} \lambda_{1 / z_{1 /, i k j} .}^{i} .
\end{aligned}
$$

Multiply by $\eta_{2}^{\alpha}$ (the unit normal to $S_{2}$ ), sum for $\alpha$ and we have

$$
\begin{aligned}
& b_{2 / k j}=b_{1 / k j} \eta_{1}^{\alpha} \cdot \eta_{2}^{\alpha}+\rho_{1 /, k j} \lambda_{1 / z_{1 /, i} \cdot \eta_{2}^{\alpha}}^{i}+\rho_{1 /, k} \lambda_{1 /, j}^{i} \underset{1 /, i}{\alpha} \cdot \eta_{2}^{\alpha} \\
& +\rho_{1 /, k} \lambda_{1 / 1}^{i} b_{1 / i j} \eta_{1}^{\alpha} \cdot \eta_{2}^{\alpha}+\rho_{1 /, j} \lambda_{1 /, k}^{i} z_{1 /, i}^{\alpha} \cdot \eta_{2}^{\alpha}+\rho_{1} \lambda_{1 /, k j z_{1 /, i} \cdot \eta_{2}^{\alpha}}^{\alpha} \\
& +\rho_{1} \lambda_{1 /, k}^{i} b_{1 / i j} \eta_{1}^{\alpha} \cdot \eta_{2}^{\alpha}+\rho_{1 /, j} \lambda_{1 / 1}^{i} b_{1 / i k} \eta_{1}^{\alpha} \cdot \eta_{2}^{\alpha}+\rho_{1} \lambda_{1 /, j}^{i} b_{1 / i k} \eta_{1}^{\alpha} \cdot \eta_{2}^{\alpha} \\
& +\rho_{1} \lambda_{1 / 1 / i k, j}^{i} \eta_{1}^{\alpha} \cdot{ }^{\alpha} \eta_{2}^{\alpha}+b_{1 / i k} \rho_{1} \lambda_{1 / \eta_{1 /, j}}^{i}{ }^{\alpha} \eta_{2}^{\alpha} \text {. }
\end{aligned}
$$

In the future, since unless otherwise stated covariant differentiation is with respect to the fundamental tensor of $S_{1}$, we shall note the covariant derivative of $z_{2 /, k}^{\alpha}$ by $z_{2 /, k j}^{\alpha}$.

We evaluate $z_{1 /, i}^{\alpha} \cdot \eta_{2}^{\alpha}$ as follows.

We differentiate (3.5) covariantly giving

$$
\underset{z_{1 /, i}}{\alpha}=z_{2 /, i}^{\alpha}+\rho_{2 /, i} \lambda_{2 / z_{2 /, s}}^{\alpha}+\rho_{2} \lambda_{2 /, i}^{s} \underset{2 /, s}{\alpha}+\rho_{2} \lambda_{2 / z_{2 /, s i}}^{\alpha} \underset{\alpha}{\alpha}
$$

Multiply by $\eta_{2}^{\alpha}$ and sum for $\alpha$, giving

$$
z_{1 /, i}^{\alpha} \stackrel{\alpha}{\alpha}=\rho_{2} \lambda_{2 /}^{s} b_{2 / s i} \text {. }
$$

Substituting this value for $z_{1 /, i}^{\alpha} \cdot \eta_{2}^{\alpha}$ in (3.10) we have

$$
\begin{aligned}
b_{2 / k j}= & \left(\eta_{1}^{\alpha} \cdot \eta_{2}^{\alpha}\right)\left(b_{1 / k j}+b_{1 / i j} \rho_{1 /, k} \lambda_{1 /}^{i}+b_{1 / i j} \rho_{1} \lambda_{1 /, k}^{i}+b_{1 / i k} \rho_{1 /, j} \lambda_{1 /}^{i}\right. \\
& \left.+b_{1 / i k} \rho_{1} \lambda_{1 /, j}^{i}+b_{1 / i k, j} \rho_{1} \lambda_{1 /}^{i}\right)+\rho_{1} \lambda_{1 /}^{i} b_{1 / i k} \eta_{1 /, j}^{\alpha} \cdot \eta_{2}^{\alpha} \\
& +\rho_{1 /, k} \lambda_{1 /, j \rho_{2}}^{i} \lambda_{2 /}^{s} b_{2 / s i}+\rho_{1 /, j} \lambda_{1 /, k}^{i} \rho_{2} \lambda_{2 / /}^{s} b_{2 / s i} \\
& +\rho_{1} \lambda_{1 /, k j}^{i} \rho_{2} \lambda_{2 /}^{s} b_{2 / s i} .
\end{aligned}
$$

Since the asymptotic lines on $S_{1}$ and $S_{2}$ are to correspond we have

${ }^{2}$ L. P. Eisenhart, Riemannian Geometry, 1926, Equation 56.2, p. 189.

${ }^{3}$ Ibid., Equation 56.3, p. 189. 


$$
b_{2 / i s}=\mu b_{1 / i s} .
$$

We determine $\mu$ as follows. Differentiate the second of $\left(3.6^{\prime}\right)$ covariantly, giving

$$
\lambda_{1 /, j}^{i} z_{1 /, i}^{\alpha}+\lambda_{1 / z_{1 /, i j}}^{i}+e\left(\lambda_{2 /, j}^{i} z_{2 /, i}^{\alpha}+\lambda_{2 / z_{2 /, i j}}^{i} \alpha=0 .\right.
$$

Multiply by $\eta_{2}^{\alpha}$, sum for $\alpha$ and use (3.12). This becomes

$$
\lambda_{1 / /}^{i} b_{1 / i j}\left(\eta_{1}^{\alpha} \cdot \eta_{2}^{\alpha}\right)+\rho_{2} \lambda_{1 /, j}^{i} \lambda_{2 /}^{k} b_{2 / k i}+e \lambda_{2 / 2}^{i} b_{2 / i j}=0 .
$$

Now multiply by $\lambda_{1 /}^{j}$ and sum for $j$ giving

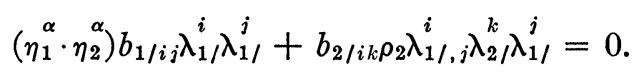

But since $b_{2 / i k}=\mu b_{1 / i k}$ we have

$$
\mu=-\frac{\left(\eta_{1}^{\alpha} \cdot \eta_{2}^{\alpha}\right) b_{1 / i j} \lambda_{1}^{i} \lambda_{1 /}^{j}}{\rho_{2} b_{1 / i k} \lambda_{1 /, j}^{i} \lambda_{2 /}^{k} \lambda_{1 /}^{j}} .
$$

Substituting in (3.13) and using the fact that ${ }^{3}$

$$
\eta_{1 /, j}^{\alpha}=-b_{1 / e j} g^{e m \alpha} z_{1 /, m}^{\alpha}
$$

we have

$$
\begin{aligned}
& \rho_{2} b_{i s} \lambda_{2 / 2}^{s} \lambda_{1 /, h}^{i} \lambda_{1 /}^{h}\left[b_{k j}+\rho_{1 /, k} \lambda_{1 /}^{i} b_{i j}+\rho_{1} \lambda_{1 /, k}^{i} b_{i j}+\rho_{1 /, \lambda_{1 /}} \lambda_{1 /}^{i} b_{i k}\right. \\
& \left.\quad+\rho_{1} \lambda_{1 /, j}^{i} b_{i k}+\rho_{1} \lambda_{1 /}^{i} b_{i k, j}\right]+b_{h r} \lambda_{1 / 1}^{h} \lambda_{1 /}^{r}\left[b_{k j}-\rho_{1 /, k} \lambda_{1 /, j}^{i} \rho_{2} \lambda_{2 /}^{s} b_{i s}\right. \\
& \quad-\rho_{1} \rho_{2} \lambda_{1 /, k j}^{i} \lambda_{2 /}^{s} b_{i s}+b_{i k} \rho_{1} \rho_{2} \lambda_{1 /}^{i} b_{e j} g^{e m} b_{m s} \lambda_{2 /}^{s} \\
& \left.\quad-\rho_{1 /, j} \lambda_{1 /, k}^{i} \rho_{2} \lambda_{2 /}^{s} b_{i s}\right]=0
\end{aligned}
$$

where the $b$ 's are all those of $S_{1}$.

To evaluate $\rho_{1}$, multiply (3.16) by $\lambda_{2 /}^{j}$ and sum for $j$, giving, by

$$
\rho_{1} b_{1 / k i} \lambda_{2 /}^{k} \lambda_{1 /, j}^{i} \lambda_{2 /}^{j}+\lambda_{2 /}^{i} \lambda_{2 /}^{j} b_{1 / i j}=0 .
$$

Similarly, we have

$$
\rho_{2} b_{1 / k i} \lambda_{1 / 1}^{k} \lambda_{2 /, j}^{i} \lambda_{1 /}^{j}+\lambda_{1 / \lambda_{1 /}}^{i} b_{1 / i j}^{j}=0
$$

where $\lambda_{2 /, j}^{i}$ is with respect to $\bar{g}_{i j}$ of $S_{2}$. (It should be remarked that $\rho_{2}$ may be expressed entirely in terms of elements of $S_{1}$ by means by (3.6) and (3.7) and differentiation.)

Equations (3.19) with $\rho_{1}$ and $\rho_{2}$ determined by (3.20) and (3.21), respectively, constitute the condition that must obtain in order for 
the tangents to the curves of direction $\lambda_{1 /}^{i}$ on $S_{1}$ to form a $W$-congruence. An equation similar to (3.19) obtains for the direction $\lambda_{2 /}^{j}$. These two equations must hold in order for the net with directions $\lambda_{1 /}^{i}$ and $\lambda_{2 /}^{j}$ to be an $R$ net.

In particular we consider the case where $\lambda_{1 /}^{i}$ and $\lambda_{2 /}^{j}$ are tangent to the $u$ and $v$ parametric curves, respectively. Then ${ }^{4}$

$$
\begin{array}{rlrl}
\lambda_{1}^{1} & =1, & & b_{11}=D, \\
\lambda_{1}^{2} & =0, & & b_{12}=b_{21}=D^{\prime}=0, \\
\lambda_{2}^{1} & =0, & & b_{22}=D^{\prime \prime}, \\
\lambda_{2}^{2} & =1, &
\end{array}
$$

and it is easily shown that

$$
\lambda_{a /, j}^{i}=\left\{\begin{array}{c}
i \\
a j
\end{array}\right\}
$$

for any fixed $i, a, j$, which is an interesting interpretation of the Christoffel symbols in this case.

In this case (3.19) reduces to

$$
2 \frac{\partial}{\partial v}\left\{\begin{array}{c}
2 \\
12
\end{array}\right\}=\frac{\partial}{\partial u}\left(\left\{\begin{array}{c}
2 \\
22
\end{array}\right\}-\frac{D^{\prime \prime}}{D}\left\{\begin{array}{c}
2 \\
11
\end{array}\right\}\right),
$$

the equation obtained by Eisenhart. ${ }^{5}$

The equation similar to $(3.19)$ reduces to

$$
2 \frac{\partial}{\partial u}\left\{\begin{array}{c}
1 \\
12
\end{array}\right\}=\frac{\partial}{\partial v}\left(\left\{\begin{array}{c}
1 \\
11
\end{array}\right\}-\frac{D}{D^{\prime \prime}}\left\{\begin{array}{c}
1 \\
22
\end{array}\right\}\right)
$$

and these two equations constitute the condition that the parametric curves of a surface $S$ form an $R$ net.

Amherst College

${ }^{4}$ L. P. Eisenhart, Differential Geometry, 1909, p. 115.

${ }^{5}$ L. P. Eisenhart, Transformation of Surfaces, 1923, p. 106. 